\title{
LINGUISTIC LAWS IN CHIMPANZEE GESTURAL COMMUNICATION
}

Heesen, Raphaela ${ }^{* 1,2}$, Hobaiter, Catherine ${ }^{3}$, Ferrer-i-Cancho, Ramon ${ }^{4}$, and Semple, Stuart $^{2}$

*Corresponding Author: raphaela.heesen @unine.ch

${ }^{1}$ Institute of Work and Organisational Psychology, University of Neuchâtel, Neuchâtel,

Switzerland

${ }^{2}$ Department of Life Sciences, University of Roehampton, London, UK

${ }^{3}$ School of Psychology and Neuroscience, University of St Andrews, St

Andrews, UK

${ }^{4}$ Complexity \& Quantitative Linguistics Lab, Departament de Ciències de la Computació, Universitat Politècnica de Catalunya, Barcelona, Catalonia

Information theory predicts that signals, as vehicles for information transfer, should be efficient in design (Shannon \& Weaver, 1962); they are required to be explicit enough to be understood through a noisy medium, but should otherwise minimize costs for the sender (Bradbury \& Vehrencamp, 1998). One way to achieve efficiency in communication systems is through Zipf's law of abbreviation (ZLA), namely to assign shorter signals to more frequent elements and longer signals to less frequent ones (Zipf, 1949).

ZLA arises from "compression" - a mathematical principle promoting efficiency in natural and artificial communications systems (Cover \& Thomas, 2006). Compression has been explored rigorously in human language, where ZLA has been shown to arise from a compromise between accuracy and efficiency (Kanwal, Smith, Culbertson, \& Kirby, 2017). Beyond ZLA, compression is also linked to Menzerath's law (ML), stating that, the larger the construct, the smaller the size of its constituents (Menzerath, 1954). Both laws have been detected across a wide range of languages (Altmann, 1980; Bentz \& Ferrer-i-Cancho, 2016), in non-human communication systems (Ferrer-i-Cancho, HernándezFernández, Lusseau, Agoramoorthy, Hsu, et al., 2013) and at the molecular level - in genes, genomes and proteins (Ferrer-i-Cancho, Forns, Hernández-Fernández, 
Bel-Enguix, \& Baixeries, 2013; Ferrer-i-Cancho, 2013; Li, 2012; Shahzad, Mittenthal, \& Caetano-Anollés, 2015).

In animal communication, patterns consistent with ZLA, i.e. a negative relationship between signal magnitude and frequency of use, have been detected in the vocal repertoire of Formosan macaques (Semple, Hsu, \& Agoramoorthy, 2010), common marmosets (Ferrer-i-Cancho \& Hernández-Fernández, 2013), bats (Luo, Jiang, Liu, Wang, Lin, et al., 2013), and in the non-vocal behavioral repertoire of dolphins (Ferrer-i-Cancho \& Lusseau, 2009). Patterns consistent with ML, i.e. an inverse relationship between construct and constituent size, have been reported for vocal sequences of geladas (Gustison, Semple, Ferrer-i-Cancho, \& Bergman, 2016) and chimpanzees (Fedurek, Zuberbühler, \& Semple, 2017). To assess the true breadth of linguistic laws, however, compression research needs to be expanded to hitherto untested modes of communication. Gestural communication, as a key signaling mode in anthropoid primates (Hobaiter \& Byrne, 2011), represents a powerful model to test linguistic laws in non-vocal communication systems beyond humans. Here, we analyzed the duration of gestures and gesture sequences given in the context of social play by wild chimpanzees, living on one community at Budongo Forest Reserve, Uganda. Gestures and sequences were recorded from animals of all age classes.

Our analyses provide the first evidence for compression in the form of linguistic laws in animal gestural communication. Although we did not initially find evidence for ZLA - i.e. an inverse relationship between gesture duration and frequency - in the overall play gesture repertoire, we found agreement with ZLA when analyzing specific gesture subsets listed according to their total duration, $D$ ( $D$ is the product of frequency of use of a gesture type and its mean duration). In particular, we found ZLA in subsets of gestures of low $D$, suggesting that compression has acted by reducing both mean duration and frequency of use. Moreover, we found a negative relationship between number of gestures in a sequence and mean duration of the constituent gestures - consistent with ML. We conclude that coding efficiency is a property shared not only between chimpanzee and human communication systems - but also across diverse taxa and modalities; it is possible that these systems may have converged to similar patterns due to similar evolutionary pressures, namely selection for least effort for sender and receiver.

\section{Acknowledgements}

RFC was supported by the grant TIN2017-89244-R from MINECO. 


\section{References}

Altmann, G. (1980). Prolegomena to Menzerath's law. Glottometrika, 2, 1-10.

Bentz, C., \& Ferrer-i-Cancho, R. (2016). Zipf's law of abbreviation as a language universal. Capturing Phylogenetic Algorithms for Linguistics. Presented at the Lorentz Center Workshop, Leiden, October 2015.

Bradbury, J. W., \& Vehrencamp, S. L. (1998). Animal communication. Massachusetts: Sinauer.

Cover, T., \& Thomas, J. (2006). Elements of information theory (Vol. 2). New York: Wiley.

Fedurek, P., Zuberbühler, K., \& Semple, S. (2017). Trade-offs in the production of animal vocal sequences: insights from the structure of wild chimpanzee pant hoots. Frontiers in Zoology, 14(1), 50. https://doi.org/10.1186/s12983-017-0235-8

Ferrer-i-Cancho, R., Forns, N., Hernández-Fernández, A., Bel-Enguix, G., \& Baixeries, J. (2013). The challenges of statistical patterns of language: The case of Menzerath's law in genomes. Complexity, 18. https://doi.org/10.1002/cplx.21429

Ferrer-i-Cancho, R., \& Hernández-Fernández, A. (2013). The failure of the law of brevity in two New World primates. Statistical caveats. Glottotheory, 4, 44-55. https://doi.org/10.1524/glot.2013.0004

Ferrer-i-Cancho, R., Hernández-Fernández, A., Lusseau, D., Agoramoorthy, G., Hsu, M. J., \& Semple, S. (2013). Compression as a universal principle of animal behavior. Cognitive Science, 37, 1565-1578. https://doi.org/10.1111/cogs.12061

Ferrer-i-Cancho, R., \& Lusseau, D. (2009). Efficient coding in dolphin surface behavioral patterns. Complexity, 14, 23-25. https://doi.org/10.1002/cplx.20266

Gustison, M. L., Semple, S., Ferrer-i-Cancho, R., \& Bergman, T. J. (2016). Gelada vocal sequences follow Menzerath's linguistic law. Proceedings of the National Academy of Sciences, 113, E2750-E2758. https://doi.org/10.1073/pnas.1522072113

Hobaiter, C., \& Byrne, R. W. (2011). The gestural repertoire of the wild chimpanzee. Animal Cognition, 14(5), 745-767. https://doi.org/10.1007/s10071-011-0409-2

Kanwal, J., Smith, K., Culbertson, J., \& Kirby, S. (2017). Zipf's law of abbreviation and the principle of least effort: Language users optimise a miniature lexicon for efficient communication, 165, 45-52. http://dx.doi.org/10.1016/j.cognition.2017.05.001

Li, W. (2012). Menzerath's law at the gene-exon level in the human genome. Complexity, 17. https://doi.org/10.1002/cplx.20398

Luo, B., Jiang, T., Liu, Y., Wang, J., Lin, A., Wei, X., \& Feng, J. (2013). Brevity is prevalent in bat short-range communication. Journal of 
Comparative Physiology A, 199, 325-333.

https://doi.org/10.1007/s00359-013-0793-y

Menzerath, P. (1954). Die Architektonik des Deutschen Wortschatzes. Bonn: Dümmler.

Semple, S., Hsu, M. J., \& Agoramoorthy, G. (2010). Efficiency of coding in macaque vocal communication. Biology Letters, 6, 469-471. https://doi.org/10.1098/rsbl.2009.1062

Shahzad, K., Mittenthal, J. E., \& Caetano-Anollés, G. (2015). The organization of domains in proteins obeys Menzerath-Altmann's law of language. BMC Systems Biology, 9, 44. https://doi.org/10.1186/s12918-015-01929

Shannon, C., \& Weaver, W. (1962). The mathematical theory of communication. Urbana: The University of Illinois Press.

Zipf, G. (1949). Human behaviour and the principle of least effort. Cambridge: MA: Addison Wesley. 